\title{
Weighted Bipartite Network Projection for Personalized Recommendations
}

\author{
Jing Wang, Fengjing Shao, Shunyao Wu, Rencheng Sun, and Ran Li
}

\begin{abstract}
To reduce the difficulty of personalized recommendations, the traditional network-based method constructed bipartite networks with stronger links (higher ratings). However, weaker links and link weights were almost ignored. Although the existing method effectively mined users' preferences, it was impossible to catch users' disgusts. Therefore, this paper proposed a novel method to effectively discover users' preferences and disgusts. Experimental results on the MovieLens dataset demonstrated that the proposed method was much more superior to the baseline method under the diversity index.
\end{abstract}

Index Terms-Personalized recommendations, weighted bipartite network, users' preferences, users' disgusts, diversity.

\section{INTRODUCTION}

With the rapid development of the Internet, the growing amounts of data had gone beyond our processing capacities, and we had entered into the era of data explosion. The overwhelming information brought the information overload problem, which had also become an urgent problem to mine valuable information from the huge data. As an important way to filter information, personalized recommendations had attracted more and more attention. Personalized recommendations aimed to discover users' preferences for recommending objects by utilizing the historical activities and personal profiles.

Currently, the recommendation methods were mainly divided into three classes, collaborative filtering methods, content-based methods and network-based methods [1], [2]. Collaborative filtering methods [3]-[6] aimed to identify some users whose preferences were similar to a given user, and then recommended objects they chosen to the target user.

Nevertheless, some limitations existed in collaborative filtering method, such as cold-start, data sparse and scalability [1]. Content-based methods [7], [8] tried to recommend the objects similar with those chose by a target user. Content-based methods overcame the cold-start and data sparse problems. However, it was difficult to predict new interests because this method was completely dependent on features of objects selected in the past. Meanwhile, Content-based methods could not recommend objects to new users without historical data.

Recently, to avoid false attribute information of the user or the object, network-based methods had been widespread

Manuscript received August 21, 2015; revised January 21, 2015.

Jing Wang, Fengjing Shao, Shunyao Wu, Rencheng Sun, and Ran Li are with Qingdao University, Shandong, China (e-mail: sfj@qdu.edu.cn). concerned by researchers. Network-based methods abstracted users and objects into nodes and abstracted user-object relationships into edges. During the recommended process, the useful information was hidden in the relationships between users and objects. Aggarwal et al. [9] firstly proposed a network-based method based on collaborative filtering mechanics, and the simulation results validated the effectiveness and efficient of the method. To highlight a possible way for the better solution of personalized recommendation, Zhou et al. [10] proposed an effective projection method on the bipartite network, and designed a network-based inference algorithm for recommendations. To further improve the algorithm accuracy and made recommendation more diversified, Zhou et al. [11] introduced a free parameter to regulate the initial configuration of resource, so as to decrease the initial resource of popular objects.

However, network-based method only constructed bipartite networks with strong links (high user-object ratings) specified by a given threshold, but neglected low user-object ratings. To solve the above problems, some work had carried on the preliminary attempt but there were still some drawbacks. For instance, some work just used the user-object rating as the edge weight to construct weighted network. During the resource diffusion process, the resources were unequally allocated according to a proportion which was the edge's weight accounted for the total edges' weight of a given node, so as to ensure that the high-rating objects were recommended preferentially. However, ignoring weak links would lost opportunities to discover users' disgusts.

To effectively mine users' preferences and disgusts, this paper considered to differentiate the rating levels. Firstly, this paper normalized ratings by half cumulative distribution method for each user. Secondly, different impacts between weak links (i.e., lower ratings) and stronger links (i.e., higher ratings) were considered for personalized recommendations. Experimental results on MovieLens dataset demonstrated the diversity of the proposed method was much more superior to competitive methods.

\section{WEIGHTED NETWORK-BASED INFERENCE}

Bipartite networks had brought a new opportunity for personalized recommendations. A bipartite network consisted of two kinds of heterogeneous nodes, and edges just existed in heterogeneous nodes. Bipartite networks described the relationships between users and objects, such as purchase behaviors. This paper aimed to propose a novel weighted bipartite network projection method for personalized recommendations by effectively utilizing user-object ratings. 


\section{A. Rating Normalization by Half Cumulative Distribution}

The rating criteria varied from people to people. Some people were rather harshly, while some people were rather loosely. Generally, we assumed that users would choose those objects that were not only liked by many users but rated high scores. However, the original ratings could not exactly reflect users' preferences since different users had different rating criteria.

TABLE I: RATINGS FOR DIFFERENT OBJECTS BETWEEN U1 AND U2

\begin{tabular}{cllllllllll}
\hline \hline Object & $\mathrm{O}_{1}$ & $\mathrm{O}_{2}$ & $\mathrm{O}_{3}$ & $\mathrm{O}_{4}$ & $\mathrm{O}_{5}$ & $\mathrm{O}_{6}$ & $\mathrm{O}_{7}$ & $\mathrm{O}_{8}$ & $\mathrm{O}_{9}$ & $\mathrm{O}_{10}$ \\
\hline $\mathrm{U} 1$ & 1 & 2 & 3 & 3 & 3 & 4 & 4 & 4 & 4 & 5 \\
$\mathrm{U} 2$ & 1 & 1 & 2 & 2 & 2 & 3 & 3 & 4 & 4 & 5 \\
\hline \hline
\end{tabular}

Table I provided an example of two users who gave scores to 10 objects. As shown in Table I, the objects were numbered in the ascending order of preference. User U1 rated loosely compared to user U2. For example, '4' indicated a stronger preference for U2 than U1 because user U2 only assigned ' 4 ' to 2 objects while $U 1$ to 4 objects. In this case, the distribution of users' ratings could effectively recognize users' preferences. More specifically, it was important to convert the original rating into users' underlying preference likelihood. Therefore, this paper normalized users' ratings by making full use of the half cumulative distribution method [12]. The formula of normalizing ratings was as follows.

$$
w_{i j}^{\prime}=\left\{\begin{array}{cc}
P\left(R \leq R_{u_{i}}\left(o_{j}\right) \mid u_{i}\right)-P\left(R=R_{u_{i}}\left(o_{j}\right) \mid u_{i}\right) / 2 & R>0 \\
0 & R=0
\end{array}\right.
$$

Here, $R$ denoted the original ratings that the user $u_{i}$ gave a score to the object $o_{j} ; R_{u_{i}}\left(o_{j}\right)$ denoted the rating set and ranged from 1 to $5 ; P\left(R \leq R_{u_{i}}\left(o_{j}\right) \mid u_{i}\right)$ denoted the rating possibility for user $u_{i}$ to rate any objects was less than or equal to $R_{u_{i}}\left(o_{j}\right) ; P\left(R=R_{u_{i}}\left(o_{j}\right) \mid u_{i}\right)$ denoted the rating possibility for user $u_{i}$ to rate any object was equal to $R_{u_{i}}\left(o_{j}\right)$.

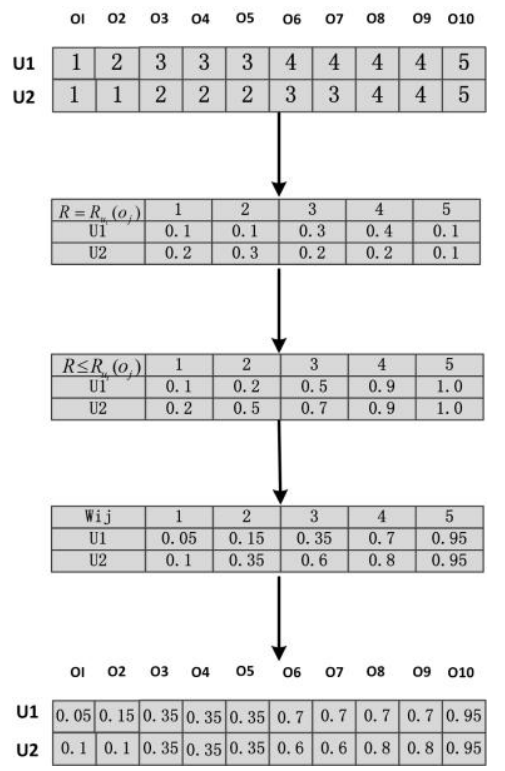

Fig. 1. The detailed steps of normalization for ratings.

To intuitively show the effect of rating normalization, Fig.
1 provided an example of normalizing users' ratings according to the half cumulative distribution method. As shown in Fig. 1, normalized score of '4' for two users (U1 and U2) were different. This example exactly showed how to mine users' tastes.

The rating normalization mapped the original ratings into the user's underlying preference likelihood. In general, a high rating indicated that a user has positive attitude on an object, while a low rating indicated that a user was likely to reject an object. Thus, we regarded the low rating as users' disgust attitude, and took both positive attitude and disgust attitude into account. It was helpful to mine the user's taste by considering the positive and the negative rating.

After normalizing ratings, the averaged preference likelihood for any user on all of the rated objects was exactly 0.5 according to the above Equation (1). Here, we proposed the following hypothesis [13]. If user $u_{i}$ had not selected a given object $o_{j}$, it could not reflect the user's preference for lacking the rating.

Based on the above hypothesis, when user $u_{i}$ had not select object $o_{j}$, we set $w_{i j}^{\prime}=0$, which indicated that user's attitude was uncertain. And when user $u_{i}$ had selected object $o_{j}$, we set $w_{i j}^{\prime}>0.5$, which indicated that the user held the positive attitude; $w_{i j}^{\prime}<0.5$ indicated user $u_{i}$ held the disgusting attitude; $w_{i j}^{\prime}=0.5$ indicated that the user's attitude was uncertain but there was a certain recommended capability, and this paper set up a relatively small tunable parameter to regulate the impact of this case.

Further, the new edge weight considering the positive and negative rating was computed by the following Equation (2).

$$
w_{i j}^{\prime \prime}=\left\{\begin{array}{cc}
w_{i j}^{\prime}-0.5+\varepsilon & 0<w_{i j}^{\prime}<1 \\
0 & w_{i j}^{\prime}=0
\end{array}\right.
$$

Here, $\varepsilon$ was a tunable parameter. It was used to distinguish $w_{i j}^{\prime}=0$ and $w_{i j}^{\prime}=0.5$.

\section{B. Weighted Bipartite Network Projection.}

Suppose there were $n$ users and $m$ objects in a recommended system, which could utilize bipartite network model to describe. Node sets in the bipartite network consisted of the object set and the user set. Denote the object set with $O=\left\{o_{1}, o_{2}, o_{3} \ldots, o_{n}\right\}$, the user set with $U=\left\{u_{1}, u_{2}, u_{3}, \ldots, u_{m}\right\} \quad, \quad$ the edge set with $E=\left\{e_{1}, e_{2}, e_{3}, \ldots, e_{l}\right\}=\left\{\left(u_{i}, o_{j}\right) \mid u_{i} \in U, o_{j} \in O\right\} \quad$ and the edge weight set with $W=\left\{w_{i j} \mid\left(u_{i}, o_{j}\right) \in E, u_{i} \in U, o_{j} \in O\right\}$. The adjacent matrix of the network was denoted by $A=\left(a_{i j}\right)_{n * m}$.

$$
a_{i j}=\left\{\begin{array}{cc}
0 & \left(u_{i}, o_{j}\right) \notin E \\
w_{i j} & \left(u_{i}, o_{j}\right) \in E
\end{array}\right.
$$

where $d\left(u_{i}\right)$ was the sum of the $i^{\text {th }}$ column of $A$, which 
stood for the sum of weights of user $u_{i}$. And $d\left(o_{j}\right)$ was the sum of the $j^{\text {th }}$ row of $A$, which stood for the sum of weights of object $o_{j}$.

Based on the resource-allocation dynamics, Zhou et al. [10] firstly designed Network-based Inference (NBI) in bipartite networks. NBI took full advantage of the user-object relationship and respectively regarded the degree of objects and the degree of users as the edge weight to equally allocate resources. And this paper was inspired by NBI. The mainly improvement was how to determine the edge weight according to the original ratings and used the edge weight to unequally allocate resources. For user $u_{i}$, the weighted network-based method started by assigning the initial resource for objects. If object $o_{j}$ had been chosen by user $u_{i}$, it would be assign one unit resource as its initial resource, otherwise zero. The initial vector of user $u_{2}$, as shown in Fig.

2 , was $\left(f\left(o_{1}\right), f\left(o_{2}\right), f\left(o_{3}\right), f\left(o_{4}\right)\right)=(0,1,1,0)$. Then the resource was redistributed between the object and the user in the weighted bipartite network. The resource-allocation process consisted of two steps.

1) The resource flowed from the object side to the user side.

The resource of object $o_{j}$ was assigned to its neighbor users according to the ratio of the edge weights. The total resources of user $u_{l}$ were as follows:

$$
g\left(u_{l}\right)=\sum_{j=1}^{n} a_{l j} f\left(o_{j}\right) / d\left(o_{j}\right)
$$

Here, $g\left(u_{l}\right)$ was the resource that user $u_{l}$ would obtain from its neighbor objects; $f\left(o_{j}\right)$ was the initial resource for object $o_{j}$.

2) The resource flowed from the user side to the object side. In a similar way, the finally resources that object $o_{j}$ obtained from its neighbor users across the whole process of allocation were as follows:

$$
f^{\prime}\left(o_{j}\right)=\sum_{l=1}^{m} a_{l j} g\left(u_{l}\right) / d\left(u_{l}\right)
$$

By plugging Equation (4) into Equation (5), the resource-allocation process was simplified as:

$$
f^{\prime}\left(o_{j}\right)=\sum_{j=1}^{n} s_{i j} f\left(o_{j}\right)
$$

Here, $s_{i j}$ denoted the object $o_{i}$ and object $o_{j}$ similarity coefficient. The formula was defined as follows:

$$
s_{i j}=\left(1 / d\left(o_{j}\right)\right) \sum_{l=1}^{m}\left(a_{i l} a_{j l}\right) / d\left(u_{l}\right)
$$

Fig. 2 provided an example of the resource-allocation process for user $u_{2}$ in the weighted bipartite network:
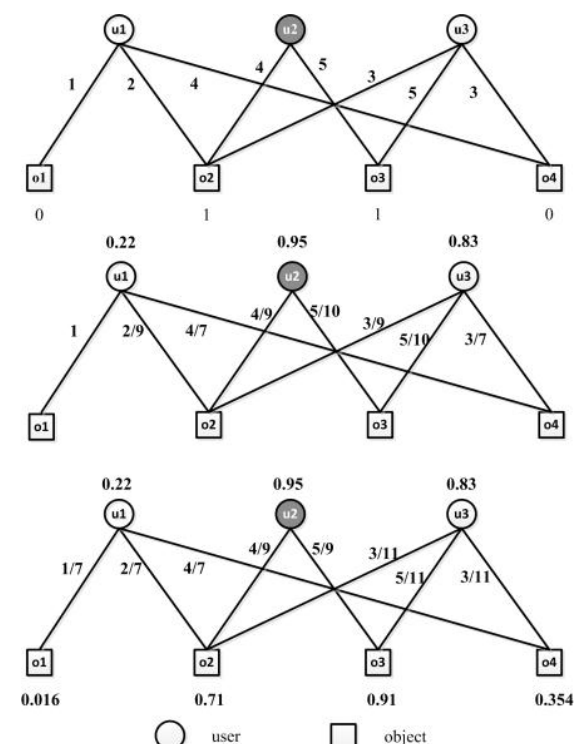

Fig. 2. Resource-allocation process in weighted bipartite network.

From the Fig. 2, the target user $u_{2}$ was filled with gray, for example. Objects that were chosen by user $u_{2}$ were distributed to one unit resource. Firstly, the resource flows from object to user, user $u_{2}$ obtained 0.95 unit resources from its neighbor object $o_{2}$ and $o_{3}$. User $u_{2}$ was more similar to user $u_{3}$ than user $u_{1}$ from Fig. 2. Then users' resources were returned to its neighbor objects again. Finally, object $o_{4}$ was priority to recommend user $u_{2}$. It was because user $u_{2}$ was similar to user $u_{3}$ and user $u_{3}$ gave a highly evaluation to object $o_{4}$.

The above method only regarded the original rating as the edge weight. Since different users had different rating criteria, the original ratings were normalized according to Equation (1) and the new edge $w_{i j}^{\prime}$ was calculated. The new edge weight set was $W^{\prime}=\left\{w_{i j}^{\prime} \mid\left(u_{i}, o_{j}\right) \in E, u_{i} \in U, o_{j} \in O\right\}$ and the new adjacent matrix of the network was denoted by $A^{\prime}=\left(a_{i j}^{\prime}\right)_{n * m}$. Here, $a_{i j}^{\prime}=w_{i j}^{\prime}$. Meanwhile, the new similarity coefficient between any two objects was derived as follows:

$$
s_{i j}^{\prime}=\left(1 / d\left(o_{j}\right)\right) \sum_{l=1}^{m}\left(a_{i l}^{\prime} a_{j l}^{\prime}\right) / d\left(u_{l}\right)
$$

Here, $a_{i l}^{\prime}$ was the normalized rating instead of the corresponding original rating.

Based on the description, we also considered the positive and negative impacts after rating normalization, and then redefined the edge weight $w_{i j}^{\prime \prime}$ by Equation (2). The latest edge weight set was $W^{\prime \prime}=\left\{w_{i j} \mid\left(u_{i}, o_{j}\right) \in E, u_{i} \in U, o_{j} \in O\right\}$ and the final adjacent matrix of the network was denoted by $A^{\prime \prime}=\left(a_{i j}^{\prime \prime}\right)_{n * m}$. Here, $a_{i j}^{\prime \prime}=w_{i j}^{\prime \prime}$. And the final resources' allocation was defined as follows:

$$
f^{\prime \prime}\left(o_{j}\right)=\sum_{j=1}^{n} s_{i j}^{\prime \prime} f\left(o_{j}\right)
$$


Here, $s_{i j}^{\prime}$ was computed by Equation (10), which denoted the similarity between any two objects

$$
s_{i j}^{\prime \prime}=\left(1 / d\left(o_{j}\right)\right) \sum_{l=1}^{m}\left(a_{i l}{ }^{\prime \prime}{ }_{j l}\right) / d\left(u_{l}\right)
$$

\section{Algorithm Description}

Algorithm1: Weighted Bipartite Network Projection Algorithms (WPNBI) Input : the adjacent matrix of user-object $A$, user $i$, the object set chose by user $i$.

Output : recommendation list $L$ for user $i$

1) Input the adjacent matrix $A$

2) Normalize the originally ratings by Equation (1) and obtain the normalized edge weight $w_{i j}^{\prime} \in[0,1)$ for each user-object rating.

3) Obtain the final edge weight $w_{i j}^{\prime \prime} \in(-0.5,0.5)$ by Equation (2)

4) Construct weighted bipartite network and the edge weight was $w_{i j}^{\prime \prime}$

5) Compute the similarity coefficient $s_{i j}^{\prime \prime}$ by Equation (10) and get the

final resource-allocate vector for user $i$ by Equation (9).

6) Get the recommendation list $L$.

Algorithm 1 provided the basic description of WPNBI algorithm. It considered not only ratings' difference for different users, but also the negative impact caused by the lower ratings. It made recommendation lists became more diverse.

According to the above description, the basic assumption that the greater the recommended value was, the higher the possibility that user liked object. For a given user, his recommendations list that included his entire unselected objects was generated by the recommended value in descending order.

\section{RESULT}

\section{A. Experiments Setup}

In this paper, the MovieLens dataset was downloaded from the website of GroupLens Research (http://www.grouplens.org). Table II listed the detailed properties of the MovieLens data.

TABLE II: DATASET PROPERTIES

\begin{tabular}{cc}
\multicolumn{2}{c}{ TABLE II: DATASET PROPERTIES } \\
\hline \hline Properties & Value \\
\hline number of users & 943 \\
number of movies & 1682 \\
rating Grade & $1-5$ \\
rating $>2$ & 82520 \\
all Ratings & 100000 \\
users' average degree & 106.1 \\
movies' average degree & 59.4 \\
\hline \hline
\end{tabular}

To evaluate the performance of proposed algorithms, this paper set up three comparative experiments. There were NBI algorithm, WNBI algorithm and SWNBI algorithm. NBI, which was proposed by Zhou et al. [10] based on a resource-allocation process, extracted the ratings greater than 2 to construct weighted bipartite network and used the user-object relationships to equally allocate resources. Based on NBI, two improved algorithm respectively named WNBI and SWNBI were derived. Different with NBI, WNBI treated user-object original ratings as edge weights to unequally allocate resources. Furthermore, SWNBI utilized the normalized ratings computed by Equation (1) as the edge weight. Thus, WNBI, SWNBI and WPNBI used all the ratings to construct weighted bipartite network. Fig. 3 demonstrated the distribution of original ratings.

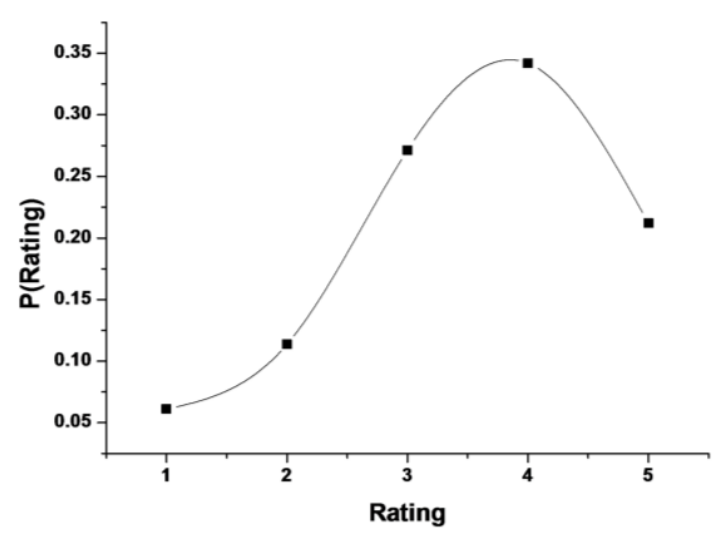

Fig. 3. The distribution of ratings before normalization.

To evaluate the performance of personalized recommendations, 10 -fold cross-validation was adopted to randomly divide the dataset into the two parts, training set and test set. For each fold, $90 \%$ of the dataset were selected as training set, while the remaining $10 \%$ as test set. In addition, the tunable parameter set to $\varepsilon=0.01$.

\section{B. Evaluation Metrics}

\section{1) Precision and recall}

Precision and Recall was typically employed to evaluate the algorithm performance, which was defined as follows:

$$
P=\frac{1}{n} \sum_{i=1}^{n} N_{r}^{i} / L
$$

Here, $N_{r}^{i}$ represented the number of objects collected by user $u_{i}$ appeared in both the testing set and recommendation list set; $L$ was the length of recommendation list.

To give a definition of Recall was as follows:

$$
R=\frac{1}{n} \sum_{i=1}^{n} N_{r}^{i} / N_{p}^{i}
$$

Here, $N_{p}^{i}$ represented the number of collected objects of user $u_{i}$ in the testing set.

\section{2) Findex}

Cleverdon C. W. [14] found that there was a negative correlation between Precision and Recall with increasing the length of recommendation list. In order to comprehensively verify the performance, Pazzani M. [15] proposed F index by simultaneously utilizing Precision and Recall. F index was 
defined as follows.

$$
F=(2 \times R \times P) /(R+P)
$$

\section{3) Diversity}

Personalized recommendation algorithms should present different of recommendation lists for different users according to their interests and habits. The average Hanming distance [11] was used to quantify the recommended diversity, $S=<H_{i j}>$, where $H_{i j}$ was defined as follows.

$$
H_{i j}=1-Q_{i j}(L) / L
$$

Here, $Q_{i j}(L)$ represented the number of overlapped objects among the recommendation lists of $u_{i}$ and $u_{j} . S$ ranged from 0 to 1 . $S=1$ denoted that all of recommendation lists were entirely different, and $S=0$ showed that all of recommendation lists were exactingly same.

\section{4) Significant test}

Significant test was used to check whether there were significantly difference between the proposed method and other comparison methods. Suppose HO hypothesis was that there was no significant difference.

For conveniently judging whether there was the difference, the $p$ value was used to quantify. Generally the $p$ was 0.05 . When $p<0.05$ represented that it rejected H0 hypothesis and accepted $\mathrm{H} 1$ hypothesis and it had significant difference, otherwise.

\section{Numerical Results}

\section{1) The comparison between WNBI and SWNBI}

In order to validate the effectiveness of the rating normalization, we conducted a comparative experiment between WNBI and SWNBI. In this experiment, the length of recommendation list $L$ was set to 20 .

TABLE III: COMPARISON OF DIFFERENT METHODS

\begin{tabular}{ccccc}
\hline \hline & Recall & Precision & F & Diversity \\
\hline NBI & 0.2461 & 0.107 & 0.149 & 0.704 \\
WNBI & 0.2383 & 0.1263 & 0.165 & 0.7183 \\
SWNBI & 0.2464 & 0.1306 & 0.1707 & 0.729 \\
\hline \hline
\end{tabular}

As shown in Table III, F and diversity of WNBI respectively outperformed NBI by $1.6 \%$ and $1.43 \%$. This indicated that the accuracy and diversity was improved by considering the ratings information. Compared to WNBI, SWNBI respectively enhanced $3.45 \%$ and $1.53 \%$ in terms of $\mathrm{F}$ and diversity. Thus, it was helpful to improve the performance by reducing the rating differences among different users.

To verify the different performance of these algorithms on accuracy and diversity was not caused by sample error. We also conducted a significant test experiment on recall, precision, F value and diversity between SWNBI and the others. The experiment results showed that there was statistical significance between SWNBI and WNBI. Results were shows in Table IV.
TABLE IV: COMPARISON OF SIGNIFICANT-TEST RESULTS

\begin{tabular}{lllll}
\hline \hline SWNBI & Recall & Precision & F & Diversity \\
\hline NBI & 0.0047 & 0.2008 & 0.1369 & 0.024 \\
WNBI & 0.0354 & 0.0131 & 0.0046 & 0.0087 \\
\hline \hline
\end{tabular}

\section{2) The comparison between WPNBI and SWNBI}

In order to mine users' disgusts from lower ratings, the comparative experiment was conducted between SWNBI and WPNBI. In this experiment, the length of recommendation list $L$ was set to 20 .

\section{TABLE V: COMPARISON BETWEEN SWNBI AND WPNBI}

\begin{tabular}{lllll}
\hline \hline & Recall & Precision & F & Diversity \\
\hline SWNBI & 0.2464 & 0.1306 & 0.1707 & 0.729 \\
WPNBI & 0.250 & 0.1326 & 0.1733 & 0.826 \\
\hline \hline
\end{tabular}

As illustrated in Table $\mathrm{V}$, the diversity of WPNBI improved significantly compared to SWNBI. This was because that users' disgust was easily found from lower ratings. It more accurately determined users' taste and provided personalized recommendations for users.

Meanwhile, the $p$ values of the significant test on recall, precision, F index and diversity were 0.0134, 0.01539, 0.00218 and 0.0048 between SWNBI and WPNBI. There was statistical significance between SWNBI and WPNBI.

\section{3) The scalability of comparison algorithms}

To verify that the performance of WPNBI were superior to other comparison methods in different recommendation list length, especially under the diversity index. The comparison experiment was used to show the scalability of different algorithms. In this experiment, the recommendation list length $L$ ranged from 10 to 100 .

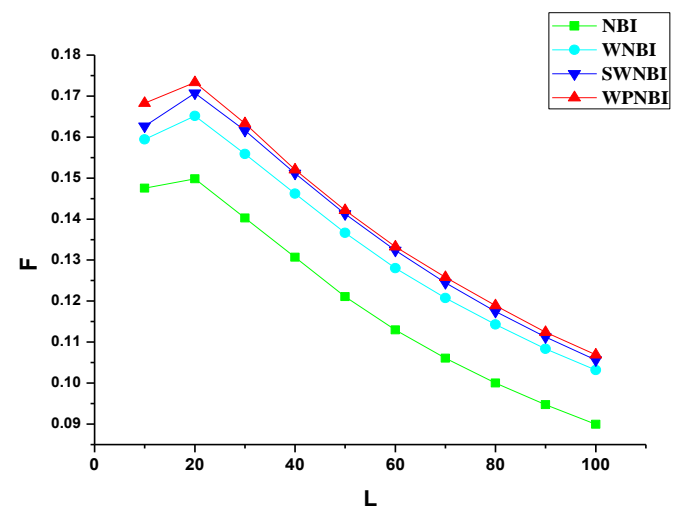

Fig. 4. The comparisons of the different methods under $\mathrm{F}$ index.

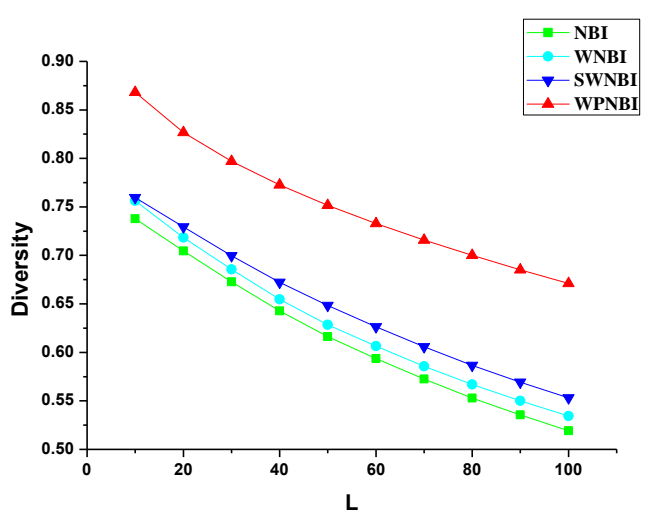

Fig. 5. The comparisons of diversity between different methods. 
There was a negative correlation between precision and recall. The $\mathrm{F}$ value was used to measure the precision of the recommendation system. As shown in Fig. 4, WPNBI performed the best in all of comparison methods under $F$ index.

Fig. 5 demonstrated the performance of different algorithms under the diversity index. Obviously, diversity decreased with increasing $L$. However, WPNBI was always superior to other algorithms. It clarified that mining users' disgusts from low ratings could effectively improve the diversity of personalized recommendations.

\section{CONCLUSION}

This paper proposed a novel recommendation algorithm to improve the diversity of the recommendation lists. We introduced the half cumulative distribution method to normalize edge weights. More specifically, this method mapped the original ratings to users' preference possibility by a normalization process. And above all, this paper took into account the negative impact caused by low ratings, which could thoroughly mine users' tastes. Experimental results showed that the diversity of the proposed algorithm was obviously superior to competitive methods.

\section{ACKNOWLEDGMENT}

This work was partially supported by the State Key Program of National Natural Science of China (No. 91130035), the National Science Foundation of Shandong Province (No. ZR2012FQ017), the National Science Foundation of Shandong Province (No. ZR2012FZ003), the National Natural Foundation (No. 41476101).

\section{REFERENCES}

[1] J. G. Liu, T. Zhou, and B. B. Wang, "Progress of the personalized recommendation systems," Progress of Nature and Science, vol. 19, no. 1, pp. 1-15, 2009.

[2] C. X. Jia, R. R. Liu, D. Sun, and B. H. Wang, "A new weighting method in network-based recommendation," Physica A: Statistical Mechanics and its Applications, vol. 387, issue 23, pp. 5887-5891, 2008.

[3] J. S. Breese, D. Heekerman, and C. Kadie, "Empirical analysis of predictive algorithms for collaborative filtering," in Proc. 14th Conf Uncertainty in Artificial Intelligence Madison, 1998, pp. 43-52.

[4] A. Nakamura and N. Abe, "Collaborative filtering using weighted majority prediction algorithms," in Proc. ICML '98 the Fifteenth International Conference on Machine Learning, 1998, pp. 395-403.

[5] H. F. Liu, Z. Hu, A. Mian, H. Tian, and X. Z. Zhu, "A new user similarity model to improve the accuracy of collaborative filtering," Knowledge-Based Systems, vol. 56, pp. 156-166, 2014.

[6] J. Zhang, Q. K. Peng, S. Q. Sun, and C. Liu, "Collaborative filtering recommendation algorithm based on user preference derived from item domain features," Physica A: Statistical Mechanics and its Applications, vol. 396, pp. 66-76, 2014.

[7] M. Balabanovic and Y. Shoham, "Fab: Content—based, collaborative recommendation," Communication of the ACM, vol. 40, no. 3, pp. 66-72, 1997.

[8] L. Pasquale, D. G. Marco, C. Musto, and N. Fedelucio, "Content-based and collaborative techniques for tag recommendation: An empirical evaluation," Journal of Intelligent Information Systems, vol. 40, no. 1, 2013.

[9] C. C. Aggarwal et al., "Horting hatches an egg: A new graph-theoretic approach to collaborative filtering," in Proc. KDD'99, San Diego, 1999, pp. 201-212.

[10] T. Zhou, J. Ren, M. Medo, and Y. C. Zhang, "Bipartite network projection and personal recommendation," Physical Review E, vol. 76, no. 4, pp. 046115, 2007.

[11] T. Zhou, L. L. Jiang, and R. Q. Su, "Effect of initial configuration on network-based recommendation," Europhys Lett., vol. 81, no. 5, pp. 8004-8008, 2008

[12] R. Jin, L. Si, C. X. Zhai, and J. Callan, "Collaborative filtering with decoupled models for preferences and ratings," $A C M$, pp. 1-58113-723-0, 2003.

[13] J. Yin, Z. S. Wang, Q. Li, and W. J. Su, "Personalized recommendation based on large-scale implicit feedback," Journal of Software, vol. 25 , no. 9, pp. 1953-1966, 2014.

[14] C. W. Cleverdon, J. Mills, and M. Kean, "Factors determining the performance of indexing systems," pp. 245-250, 1966.

[15] M. Pazzani and D. Billsus, "Learning and revising user profiles: The identification of interesting websites," Machine Learning, vol. 27, pp. 313-331, 1997.

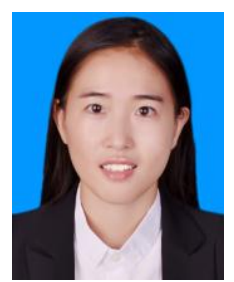

Jing Wang was born in 1992, Shandong province, China. She is a second grade postgraduate at the College of Information Engineering, Qingdao University. Currently, her research areas include data mining and complex network.

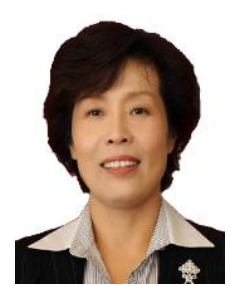

congress.

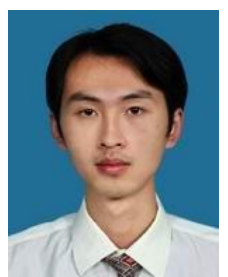

Shunyao Wu was born in 1986, Shandong province, China. He obtained the PhD degree from the College of Automation Engineering in Qingdao University in 2014. His mainly research areas are data mining and complex network. Now he is an experienced lecturer at the College of Information Engineering, Qingdao University.

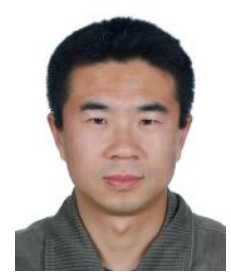

Rencheng Sun was born in 1977, Shandong province, China. He is an assistant professor and master's supervisor at the College of Information Engineering at the Qingdao University. His research areas include data mining and complex network.

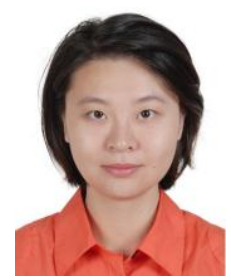

Ran Li was born in 1987, Shandong province, China She is an experienced lecturer at the College of Software Technology, Qingdao University. The mainly research area include complex network and digital media technology. 